\title{
Long non-coding RNA expression identified by microarray analysis: Candidate biomarkers in human acral lentiginous melanoma
}

\author{
HAO-ZE SHI ${ }^{1 *}$, JING-SHU XIONG $^{1 *}$, CONG-CONG XU ${ }^{1}$, WEN-BO BU ${ }^{2}$, \\ YAN WANG $^{2}$, JIAN-FANG SUN ${ }^{1}$ and HAO CHEN ${ }^{1}$ \\ Departments of ${ }^{1}$ Pathology and ${ }^{2}$ Surgery, Institute of Dermatology, Chinese Academy of Medical \\ Sciences and Peking Union Medical College, Nanjing, Jiangsu 210042, P.R. China
}

Received March 20, 2019; Accepted November 14, 2019

DOI: $10.3892 /$ ol.2019.11207

\begin{abstract}
Melanoma is a rare but fatal form of skin cancer and acral lentiginous melanoma (ALM) is one of its most common types. Long non-coding RNA (lncRNA) has emerged as a crucial molecule in the development and progression of human cancers, and several studies have revealed that lncRNAs may be associated with the pathogenesis, progression and metastasis of melanoma. To demonstrate the association between ALM and lncRNAs, microarray analysis was performed in tumor and adjacent non-tumor tissues. A total of 4,488 lncRNAs and 3,913 mRNAs were identified to be differentially expressed in these samples. Among them, 2,211 and 2,277 lncRNAs were upregulated and downregulated in the ALM samples compared with adjacent tissues, respectively. In addition, 1,191 and 2,722 mRNAs were upregulated and downregulated, respectively. Additionally, five randomly selected lncRNAs (fold-change $>2 ; \mathrm{P}<0.05$ ) were validated by reverse transcription-quantitative PCR. An lncRNA and mRNA co-expression network and competing endogenous network analysis were also constructed. In summary, the results of the present study may reveal a novel mechanism associated with the pathogenesis and malignant biological processes of ALM and indicate that lncRNAs may serve as potential targets for the treatment of ALM.
\end{abstract}

Correspondence to: Dr Hao Chen or Dr Jian-Fang Sun, Department of Pathology, Institute of Dermatology, Chinese Academy of Medical Sciences and Peking Union Medical College, 12 Jiangwangmiao Road, Nanjing, Jiangsu 210042, P.R. China E-mail: ch76ch@163.com

E-mail: fangmin5758@aliyun.com

"Contributed equally

Key words: acral lentiginous melanoma, long non-coding RNA, microarray analysis, co-expression network, competing endogenous RNA network

\section{Introduction}

Melanoma is a rare, fatal type of skin tumor, which comprises four main types: Lentigo maligna melanoma, superficial spreading melanoma (SSM), nodular melanoma and acral lentiginous melanoma (ALM) (1). ALM, which generally affects the palms and soles of patients, has a low incidence in the Caucasian population and occurs mainly in patients of an Asian and African descent; up to $75 \%$ of all patients with melanoma have ALM (1). Patients with ALM usually have a poor prognosis due to difficulties in diagnosis and ALM tends to be identified at an advanced clinical stage or with high Breslow thickness (1-4). Genomic instability and poor response to biological agents in ALM also contribute to the poor outcome. Unlike SSM, in which BRAF mutation is the most observed aberration, KIT proto-oncogene receptor tyrosine kinase is the most mutated gene in ALM; however, this has only been identified in $15 \%$ of patients (5). Therefore, identification of more specific biomarkers for ALM is necessary.

Long non-coding RNAs (lncRNAs) have been demonstrated to serve crucial roles in tumorigenesis by diverse mechanisms and at various levels; for example, lncRNAs can act as mediators to regulate gene expression, combine with proteins to form a ribonucleoprotein complex and modify histones, recruit enzymes to regulate proximal or distant genes or serve as a decoy for transcription factors $(6,7)$. Although previous studies $(8-21)$ have reported that lncRNAs including HOTAIR, MALAT1, BANCR, ANRIL, SPRY4-IT1, Llme23, UCA1, SLNCR1 and SAMMSON served oncogenic functions in the progression and metastasis of melanoma, no studies are currently available on lncRNAs specifically related to ALM, and the mechanisms of lncRNA activity in ALM are still unclear. Therefore, identification of lncRNAs in ALM may provide value for early diagnosis and improved prognosis.

The present study aimed to investigate the role of lncRNAs in the pathogenesis of ALM by performing microarray analysis of the expression patterns of lncRNAs. This study may help to clarify the function of lncRNAs in ALM and provide evidence of their therapeutic and prognostic value. 


\section{Materials and methods}

Tissue collection. A total of 12 samples, including six tumor and six adjacent non-tumor tissues, were collected in pairs from six patients with ALM (patient 1, male, 71 years; patient 2 , male, 72 years; patient 3 , female, 44 years; patient 4 , female, 66 years; female 5, female, 74 years and patient 6 , male, 55 years) between January 2017 and May 2018 at the Institute of Dermatology, Chinese Academy of Medical Sciences and Peking Union Medical College (Nanjing, China). The samples were immediately stored at $-80^{\circ} \mathrm{C}$. The study was approved by the Ethics Committee of the Institute of Dermatology, Chinese Academy of Medical Sciences and Peking Union Medical College (approval no. 2013-LC/KY-033). All participating patients gave informed consent.

RNA extraction and quality control. According to the manufacturer's protocol, total RNA was extracted using TRIzol ${ }^{\circledR}$ (Invitrogen; Thermo Fisher Scientific, Inc.). RNA quantity and quality were measured by NanoDrop ND-1000. Standard denaturing agarose gel electrophoresis (1\%) or Agilent 2100 Bioanalyzer (Agilent Technologies, Inc.) was used to assess integrity of RNA.

Microarray analysis. A total of 6 pairs of ALM and adjacent non-tumor tissues were used for the microarray assay to determine differentially expressed lncRNAs and mRNAs. Sample labeling and array hybridization were performed according to the Agilent One-Color Microarray-Based Gene Expression Analysis protocol (Agilent Technology, Inc.). Arraystar Human LncRNA Microarray V4.0, designed for the global profiling of human lncRNAs and protein-coding transcripts, was used. The hybridized arrays were washed and then scanned using Agilent Scanner G2505C (Agilent Technology, Inc.). The acquired array images were analyzed using Agilent Feature Extraction software (version 11.0.1.1). Quantile normalization and subsequent data processing were performed using the GeneSpring GX v12.1 software package (Agilent Technologies, Inc.). Following quantile normalization of the raw data, lncRNAs and mRNAs that had flags in Present or Marginal ('All Targets Value') in $\geq 5$ samples were selected for further analysis. Differentially expressed lncRNAs and mRNAs between cancerous and adjacent tissues were identified using the thresholds $\mathrm{P}<0.05$ and fold-change $>2$.

Gene ontology $(G O)$ enrichment and kyoto encyclopedia of genes and genomes (KEGG) analysis. GO analysis (http://www.geneontology.org) was performed to explore the 'biological processes', 'cellular components' and 'molecular functions' of the identified differentially expressed mRNAs, and this was performed via top GO package in R environment. Using hyper-geometric distribution, which was made using $\mathrm{R}$ language, the significance between differentially expressed genes and KEGG could be observed. Pathway analysis was performed using KEGG, Biocarta and Reactome (http://www. genome.jp/kegg/). Fisher's exact test was used to find out if the overlaps between the DE gene list and the GO annotation list were greater than what was expected by chance. The significance of each GO term and pathway association are reflected by the $\mathrm{P}$-value, and $\mathrm{P}<0.05$ was considered to indicate a statistically significant result. The $-\log _{10}(\mathrm{P})$ was used to determine the enrichment of each GO term in the differentially expressed genes and the significance of the pathway associations. A lower P-value was considered to indicate a more significant correlation. The top 10 terms of GO analysis and KEGG analysis were all characterized by $\mathrm{P}<0.05$ and false discovery rate (FDR) $<0.05$.

Reverse transcription-quantitative ( $R T-q) P C R$ validation. A total of five randomly selected lncRNAs (fold-change $>2$, $\mathrm{P}<0.05)$ were validated by RT-qPCR. These five lncRNAs belonged to the top 40 according to $\mathrm{FC}$ and its $\mathrm{P}<0.05$. All these IncRNA_levels were gold level. Gold level meant that these selected IncRNAs had been validated by specific experiments and had relevant annotation, such as transcription units, function mechanisms and subcellular localization. Following RNA extraction, SuperScript ${ }^{\mathrm{TM}}$ III Reverse Transcriptase (Invitrogen; Thermo Fisher Scientific, Inc.,) was used to synthesize cDNA according to the manufacturer's protocol. Briefly, $2 \mu \mathrm{g}$ RNA was mixed with $1 \mu 1$ lncRNA specific primer Mix, 1.6 $\mu \mathrm{l}$ dNTP Mix, and RNase free water was added to a total volume of $13.5 \mu \mathrm{l}$. The sample was placed in a water bath for $5 \mathrm{~min}$ at $65^{\circ} \mathrm{C}$ and on ice for $2 \mathrm{~min}$. Subsequently, $4 \mu 1$ 5X First-Strand Buffer, $1 \mu \mathrm{l}$ of 0.1 M DTT, $0.5 \mu 1$ RNase Inhibitor and $1 \mu$ l SuperScript III RT were added. The reaction was performed for $1 \mathrm{~min}$ at $37^{\circ} \mathrm{C}$, for $60 \mathrm{~min}$ at $50^{\circ} \mathrm{C}$ and for 15 min at $70^{\circ} \mathrm{C}$. cDNA was eventually stored at $-20^{\circ} \mathrm{C}$. PCR was performed using ViiA 7 Real-time PCR System (Applied Biosystems; Thermo Fisher Scientific, Inc.) in a total volume of $10 \mu \mathrm{l}$, including $0.5 \mu \mathrm{l}$ forward primer $(10 \mu \mathrm{M}), 0.5 \mu 1$ reverse primer $(10 \mu \mathrm{M}), 2 \mu \mathrm{l}$ cDNA, $5 \mu \mathrm{l} 2 \mathrm{X}$ Master Mix and $2 \mu \mathrm{l}$ double-distilled water. $\beta$-actin was used as an internal control. Primer sequences were as follows: $\beta$-actin forward, 5'-GTG GCCGAGGACTTTGATTG-3' and reverse, 5;-CCTGTAACA ACGCATCTCATATT-3'; NR_004845 forward, 5'-TTGGCA TACAGGTCTTTGTAGAT-3' and reverse, 5'-TTGGCATAC AGGTCTTTGTAGAT-3'; NR_026983 forward, 5'-ATCCCT GGTATTGAAGAGGTG-3' and reverse, 5'-AGATTGTTT GGGCAGTGTTAG-3'; NR_034040 forward, 5'-TGACAT CCGAATGCCATCCT-3' and reverse, 5'-GCTGCTGACAAA CAACCTGCT-3'; NR_036580 forward, 5'-AGCCCAGAT TCTCCTACCAGC-3' and reverse, 5'-CCTTCAGGTGGC TGTTTTGTAGT-3'; NR_037877 forward, 5'-ATGTTGACC ATGCAGCCAATT-3' and reverse, 5'-GTGTTTATCAGA GGTCATTTCCG-3'. The thermocycling conditions were as follows: $95^{\circ} \mathrm{C}$ for $10 \mathrm{~min}$, followed by 40 cycles of $95^{\circ} \mathrm{C}$ for $10 \mathrm{sec}$ and $60^{\circ} \mathrm{C}$ for $60 \mathrm{sec}$. The gene expression levels in tumor tissue relative to adjacent tissue were calculated as fold-change using the standard curve method (22). An unpaired t-test was used and $\mathrm{P}<0.05$ was considered to indicate a statistically significant difference.

LncRNA and mRNA co-expression (CNC) network. Using the validated lncRNAs and their target mRNAs, the CNC network was constructed by Cytoscape software (version 2.8.3; The Cytoscape Consortium) with the criterion that the Pearson correlation coefficient (PCC) of the lncRNA and mRNA correlation analysis was $\geq 0.95$. Additionally, $\mathrm{PCC} \geq 0.95$ was considered to indicate meaningfully related pair. While performing $\mathrm{CNC}$ analysis and calculating $\mathrm{PCC}$, the P-value 
and FDR were also obtained $(\mathrm{P}<0.05$ and $\mathrm{FDR}<0.05)$, which further confirmed the reliability of PCC.

Competing endogenous network analysis. Based on the hypothesis that RNA transcripts can crosstalk by competing for common microRNAs (miRNAs) and that miRNA response elements (MREs), which were the foundation of this interaction, the competing endogenous network was predicted. The prediction of such IncRNA-miRNA-mRNA interaction was based on the selected lncRNAs and related mRNAs. LncRNA/miRNA interactions were predicted by miRcode (http://www.mircode.org) and miRNA/mRNA interactions were predicted by miRanda (http://www.miranda.org) and TargetScan (http://www.targetscan.org).

\section{Results}

Differentially expressed IncRNA and mRNA profiles detected by microarray. According to the microarray expression profiling data and the filtering analysis including fold-change $\geq 2$ and $\mathrm{P}<0.05$, a total of 4,490 lncRNAs and 3,915 mRNAs were identified to be differentially expressed between the ALM and adjacent non-tumor samples. Among them, 2,211 and 2,277 lncRNAs were upregulated and downregulated in the ALM samples compared with adjacent sections, respectively. In addition, 1,191 and 2,722 mRNAs were upregulated and downregulated, respectively. The top 5 upregulated lncRNAs were T380070, ENST00000554431, T097678, GSE61474_TCONS_00183926 and NR_015399, and the top 5 downregulated lncRNAs were TCONS_00013495, T367528, ENST00000598924, T050010 and TCONS_00010140. The top 5 upregulated mRNAs were ENST00000370287, NM_005367,NM_001129826,NM_001922 and NM_000273, and the top 5 downregulated mRNAs were ENST00000433840, NM_206998, NM_014867, NM_173595 and NM_004202. The variations in the lncRNA (Fig. 1A) and mRNA (Fig. 1B) expression profiles between ALM and adjacent tissue samples were assessed by scatterplot analysis, and volcano plots were constructed to demonstrate the association between the fold-changes and the statistical significance of the differentially expressed lncRNAs (Fig. 2A) and mRNAs (Fig. 2B). The expression patterns of IncRNAs and mRNAs were also demonstrated in hierarchical clustering (Fig. 3).

GO enrichment and KEGG pathway analysis. As lncRNAs affected coding gene expression by influencing mRNAs, GO enrichment analysis of significantly differentially expressed mRNAs was performed to determine the effects of these lncRNAs. GO categories of 'biological process' (BP), 'cellular component' (CC) and 'molecular function' (MF) were analyzed to determine the gene and gene product enrichment. The results of GO enrichment analysis are presented in Fig. 4. The analysis revealed that the majority of the BPs associated with upregulated mRNAs were involved in the events of pigmentation and melanocytes, such as 'developmental pigmentation', 'melanocyte differentiation', 'pigmentation' and 'melanin metabolite biosynthetic process' (Fig. 4A). The majority of the CCs of the upregulated mRNAs were associated with 'melanosome membrane', 'pigment granule' and 'melanosome' (Fig. 4B). Terms associated with channel activity were enriched in the
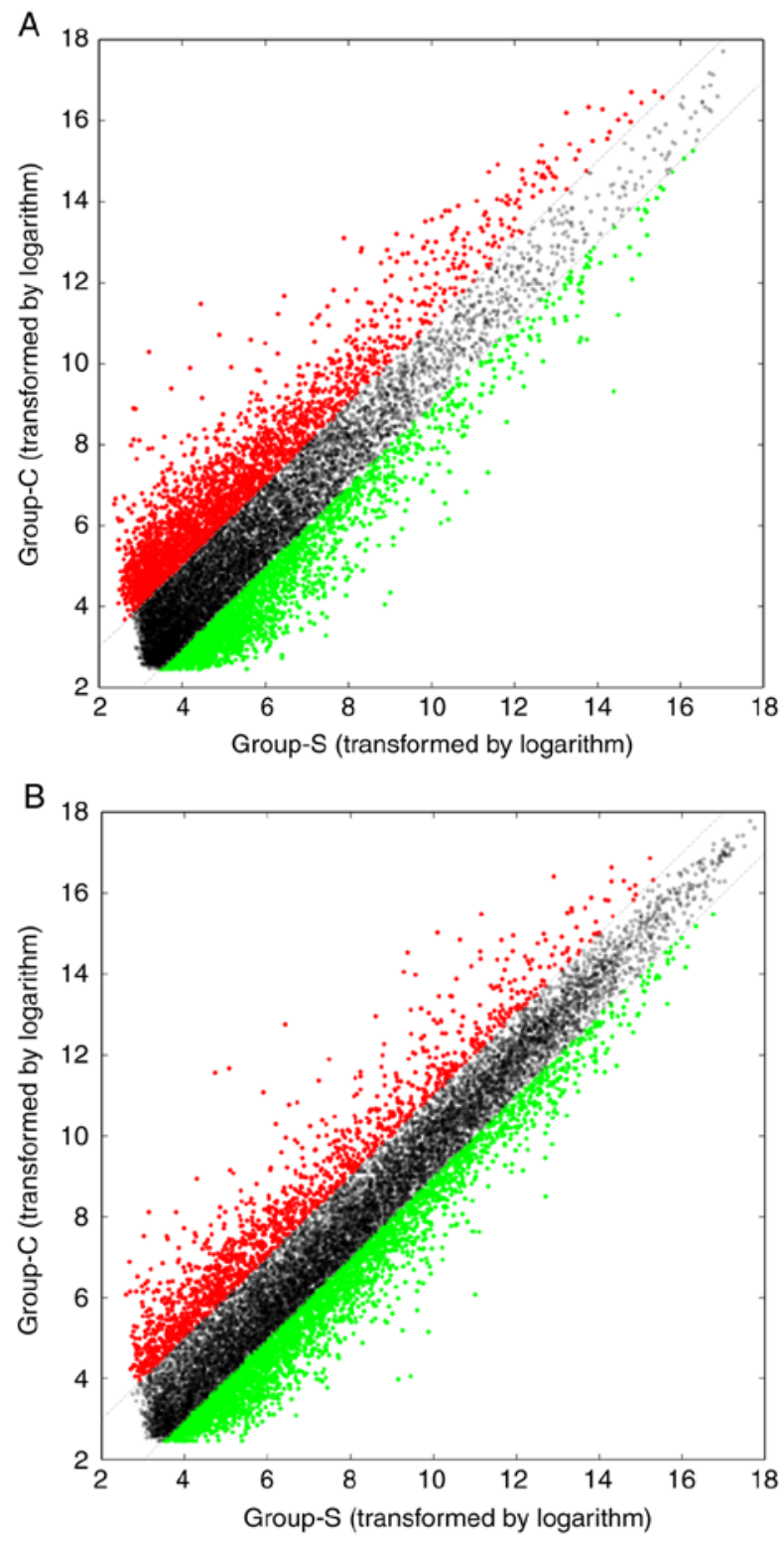

Figure 1. LncRNA and mRNA expression in ALM. (A and B) Scatterplots of (A) lncRNA and (B) mRNA expression variations between ALM and adjacent non-tumor tissues. Data points above the top line and below the bottom line indicate the IncRNA and mRNAs with fold-change $>2$ in expression between the two groups. Red points represent upregulation of differentially expressed lncRNAs and mRNAs. Green points represent downregulation of differentially expressed lncRNAs and mRNA. Black points represent none-differentially expressed lncRNAs and mRNAs. The values of the $x$ and $\mathrm{y}$ axes show the averaged normalized signal values of the group $(\log 2 \mathrm{scaled})$. Group-S, adjacent non-tumor tissues; group-C, cancerous tissues; lncRNA, long non-coding RNA; ALM, acral lentiginous melanoma.

MF category, including 'channel activity', 'cation channel activity' and 'calcium channel activity' (Fig. 4C). For the downregulated mRNAs, the majority of the enriched terms were associated with the cell junction and tissue development (Fig. 4D-F). For example, the top 3 terms in BP were 'tissue development', 'epidermis development' and 'epithelium development'. 'Cell-cell junction', 'anchoring junction' and 'cell-cell adhesion junction' were the top three terms in $\mathrm{CC}$, whereas 'cell adhesion molecule binding', 'protein binding involved in cell-cell adhesion', 'cadherin binding' and 'kinase binding or actin binding' were the most three enriched terms in MF. 
A

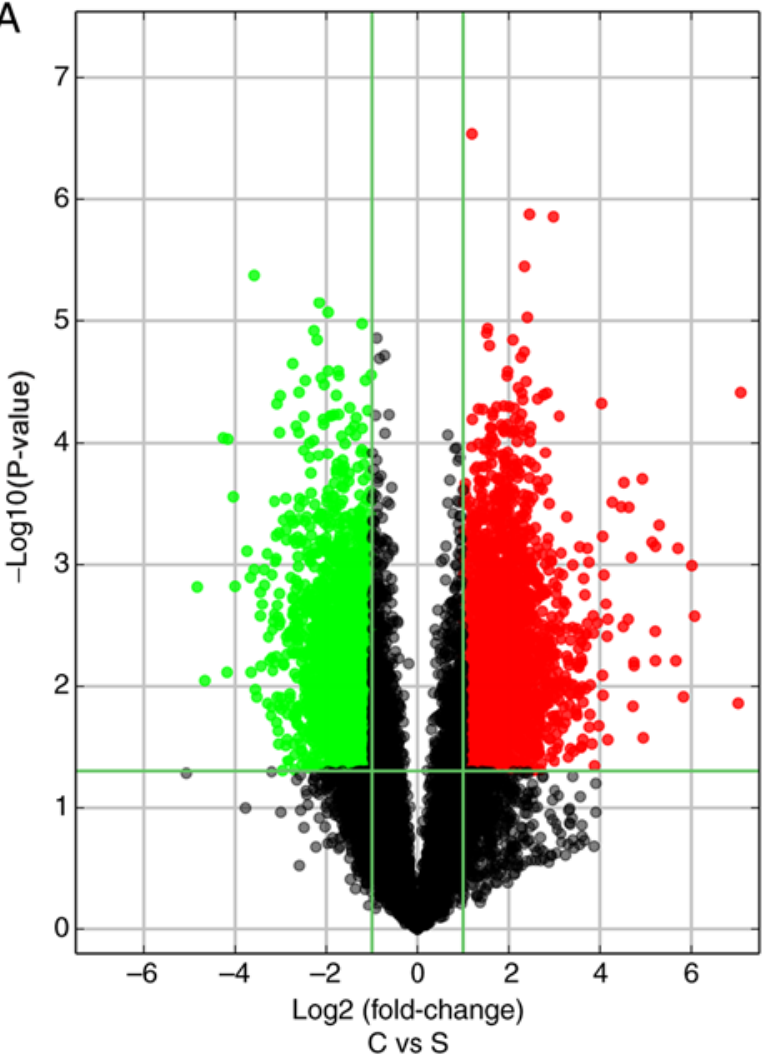

$\mathrm{B}$

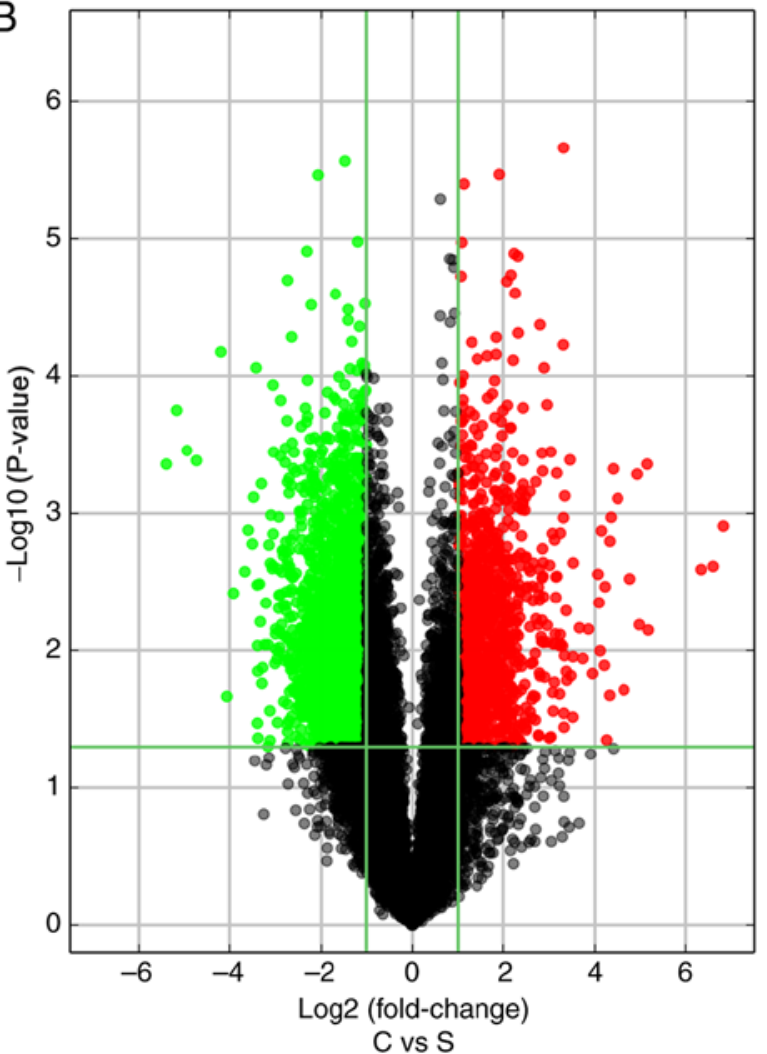

Figure 2. Volcano plots. (A and B) Differential expression of (A) lncRNAs and (B) mRNAs. Red points represent upregulation of differentially expressed lncRNAs and mRNAs. Green points represent downregulation of differentially expressed lncRNAs and mRNA. Black points represent none-differentially expressed lncRNAs and mRNAs. Two vertical lines represent the filtering standard of fold-change $\geq 2$. The term $\mathrm{C}$ vs. $\mathrm{S}$ at the bottom refers to acral lentiginous melanoma vs. adjacent tissues. Group-S, adjacent non-tumor tissues; group-C, cancerous tissues; lncRNA, long non-coding RNA.

A
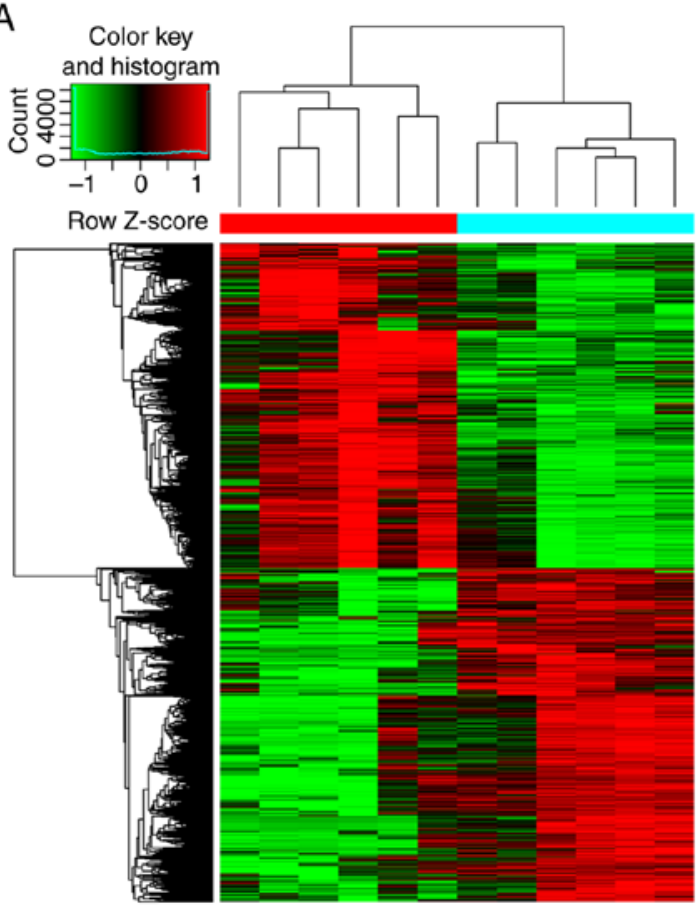

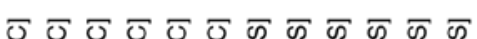

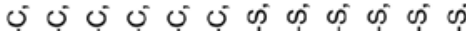

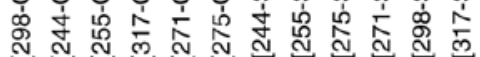

B
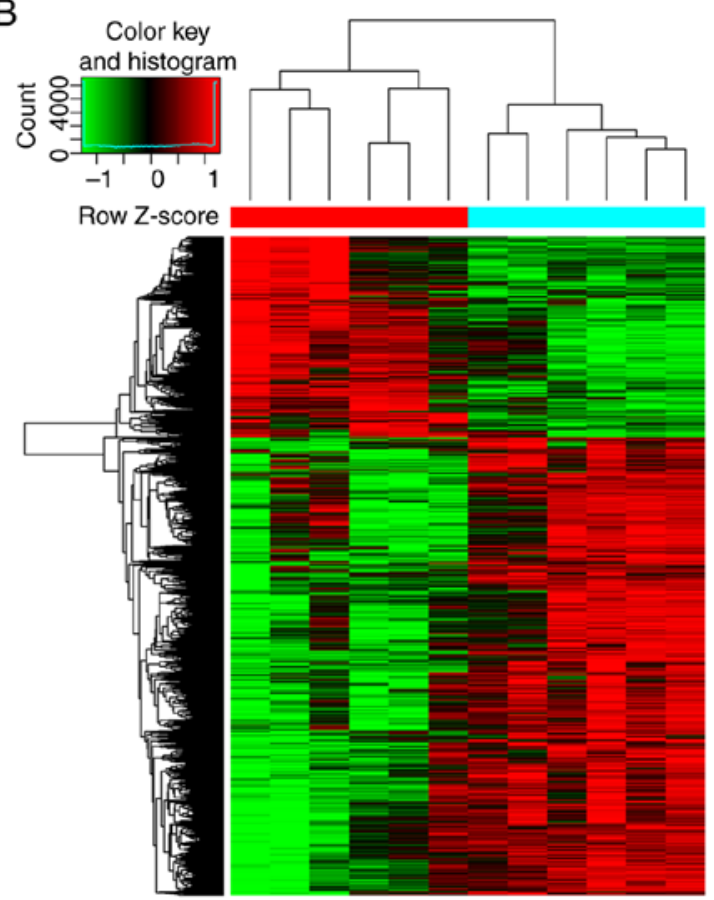

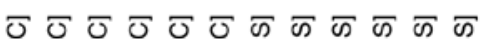

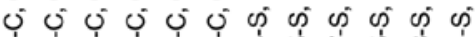

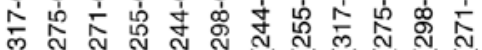

Figure 3. Hierarchical clustering. (A) LncRNAs and (B) mRNAs differentially expressed in acral lentiginous melanoma and adjacent non-tumor tissues were subjected to hierarchical clustering. Samples were divided into two groups by expression level; red indicates high relative expression and green indicates low relative expression. $\mathrm{C}$, acral lentiginous melanoma tissues; S, adjacent tissues; lncRNA, long non-coding RNA. 

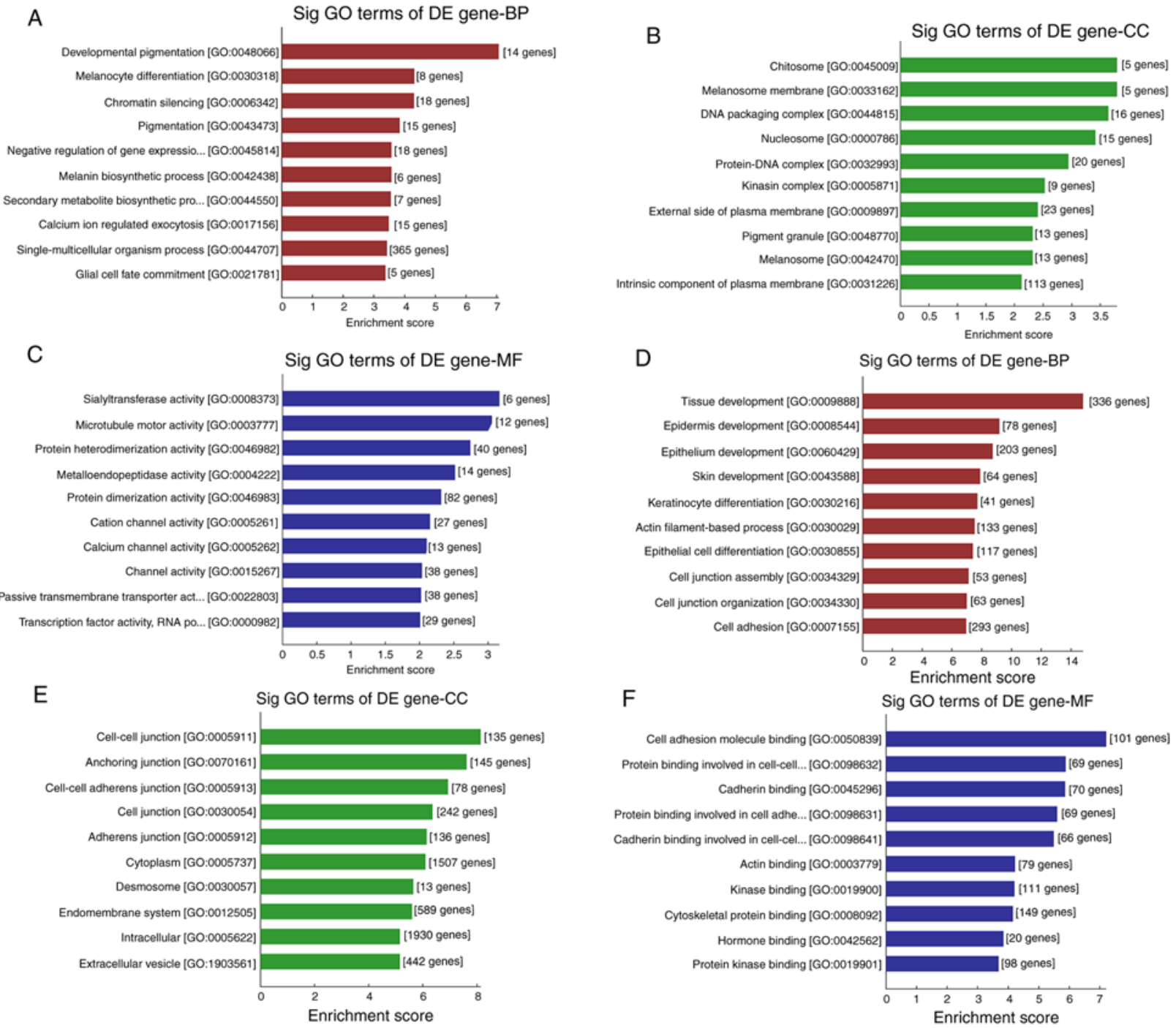

Figure 4. GO enrichment analysis. (A-F) The top 10 terms that associated with (A-C) upregulated mRNAs and (D-F) downregulated mRNAs are presented. (A and D) BP, (B and E) CC, and (C and F) MF. BP, biological process; CC, cellular component; MF, molecular function; DE, differentially expressed; GO, gene ontology.

KEGG pathway enrichment analysis results are presented in Fig. 5, including 10 pathways associated with upregulated mRNAs (Fig. 5A) and 10 pathways associated with downregulated mRNAs (Fig. 5B). These results demonstrated that the upregulated mRNAs may be involved in 'glycosphingolipid biosynthesis-ganglio series', 'transcriptional misregulation in cancer', 'toll-like receptor signaling pathway' and 'melanogenesis'. In addition, 'oxytocin signaling pathway', 'regulation of actin cytoskeleton and hippo signaling pathway', 'focal adhesion' and 'gap junction' pathways were associated with the downregulated mRNAs.

$R T-q P C R$ validation. To confirm the previous results and detect the function of IncRNAs in ALM, five randomly selected IncRNAs (Table I) were validated by RT-qPCR, including three downregulated lncRNAs NR_004845, NR_026983 and NR_034040 (Fig. 6A-C) and two upregulated lncRNAs NR_036580 and NR_037877 (Fig. 6D and E). The results were similar to those of the microarray analysis (Fig. 6). Microarray analysis and RT-qPCR demonstrated downregulated expression of NR_004845, NR_026983 and
NR_034040 and upregulated expression of NR_036580 and NR_037877. This provided reliable confirmation of IncRNA changes determined by microarray analysis.

LncRNA and mRNA CNC network, GO enrichment and KEGG analysis. Based on the five validated lncRNAs (Table I) and their target mRNAs, the co-expression network consisting of 1,064 nodes and 1,312 connections was constructed (Fig. S1). The downregulated lncRNAs NR_004845, NR_026983 and NR_034040 correlated with 391,141 and 150 mRNAs, respectively. The upregulated lncRNAs NR_036580 and NR_037877 correlated with 249 and 373 mRNAs, respectively.

GO and KEGG analysis of the CNC network were performed. The top 10 enriched GO terms in $\mathrm{BP}, \mathrm{CC}$ and $\mathrm{MF}$ are presented in Fig. 7A-C. The results revealed that, in BP, target mRNAs were enriched in 'adherens junction organization', 'pigmentation' and 'adherens junction assembly'. In CC, 'intrinsic component of membrane' and 'membrane part' were the most significantly enriched terms. In addition, terms associated with channel activity and DNA binding were enriched 
A

Sig pathway of DE gene

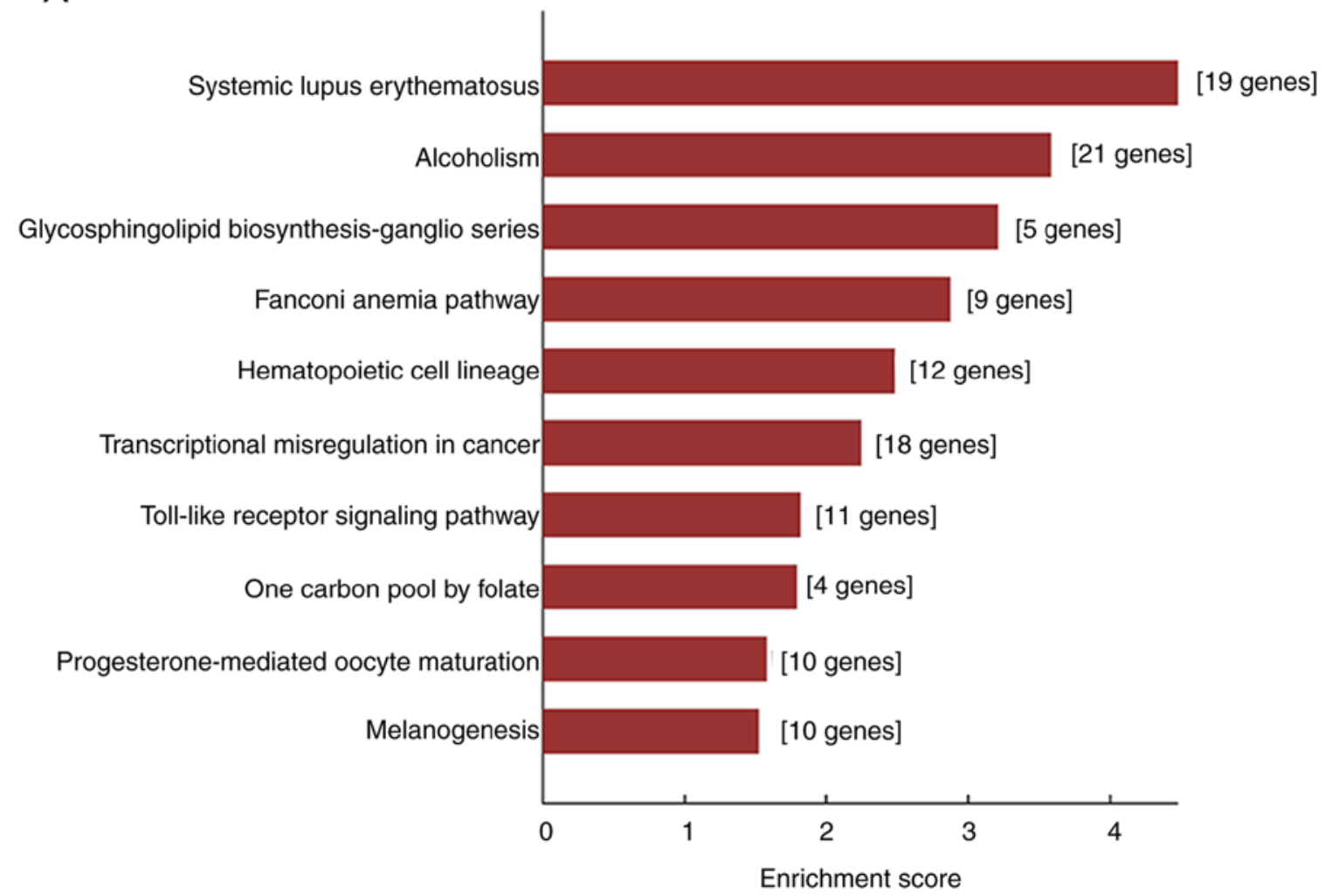

B

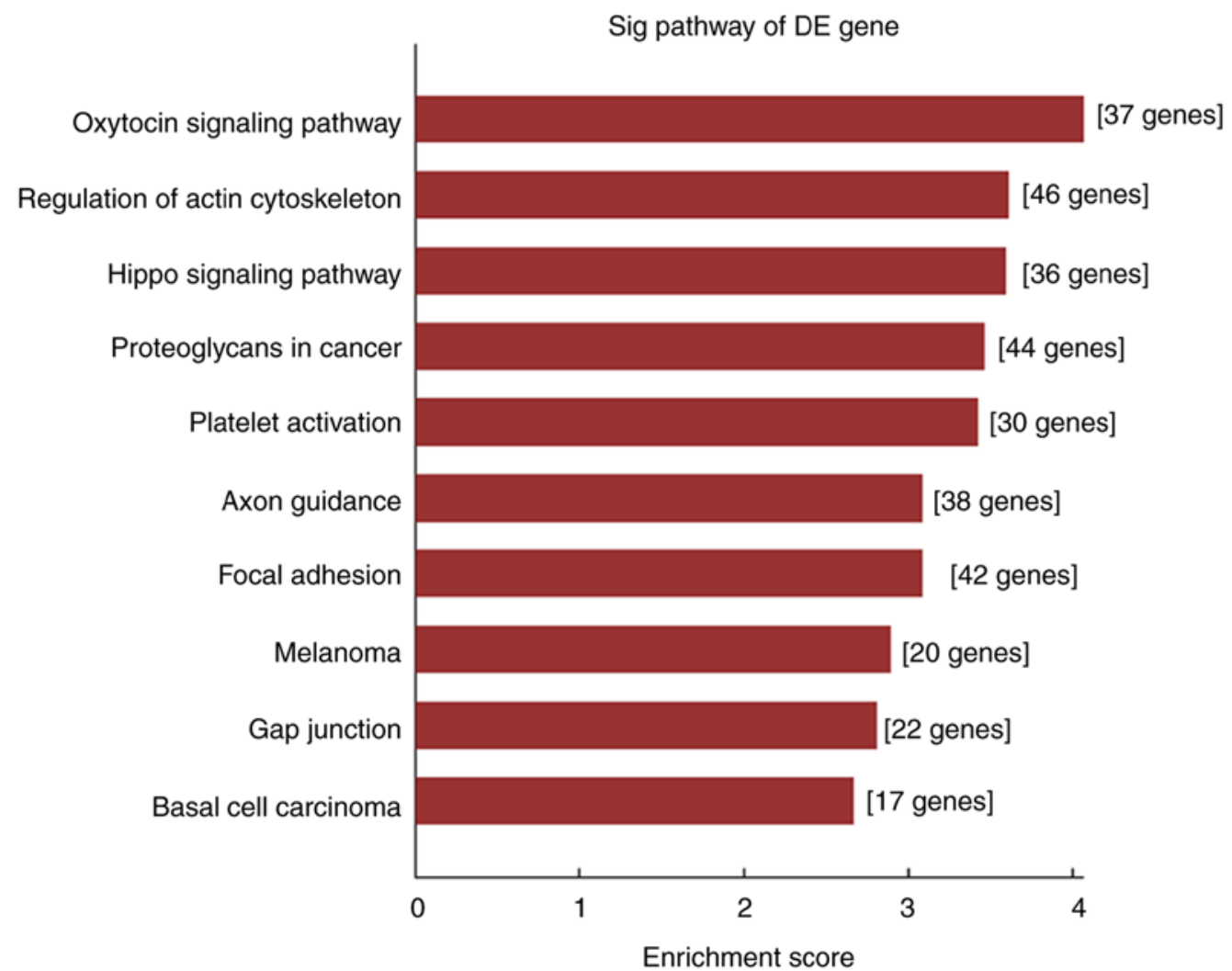

Figure 5. Kyoto Encyclopedia of Genes and Genomes pathway analysis. The top 10 pathways of (A) upregulated and (B) downregulated differentially expressed mRNAs are presented. DE, differentially expressed.

in MF. These results were in accordance with the outcome of the GO enrichment analysis of microarray results.

The KEGG analysis (Fig. 8A) demonstrated that the selected IncRNAs were associated with pathways such as 'melanogenesis', 'glycosaminoglycan biosynthesis-heparan sulfate/heparin', 'glycolysis/gluconeogenesis' and 'oxytoxin signaling pathway'. These results also revealed that the selected IncRNAs were representative. 
Table I. Five randomly selected long noncoding RNAs for reverse transcription-quantitative PCR confirmation, construction for lncRNA and mRNA co-expression network as well as competitive endogenous RNA network construction.

\begin{tabular}{llllllll}
\hline Seqname & GeneSymbol & P-value & Fold-change & Regulation & Chrom & Strand & Relationship \\
\hline NR_004845 & LOC644936 & 0.000893591 & 8.5660466 & Down & chr5 & - & Intergenic \\
NR_026983 & BTF3P11 & 0.002967842 & 8.0696294 & Down & chr13 & + & Intergenic \\
NR_034040 & LGALS8-AS1 & 0.001504141 & 16.0657987 & Down & chr1 & - & Intronic antisense \\
NR_036580 & DPP10-AS1 & 0.000199269 & 30.4236389 & Up & chr2 & - Intronic antisense \\
NR_037877 & LOC100505912 & 0.003565498 & 36.9781352 & Up & chr4 & - Intergenic \\
\hline
\end{tabular}

Fold-change $>2.0, \mathrm{P}<0.05$.
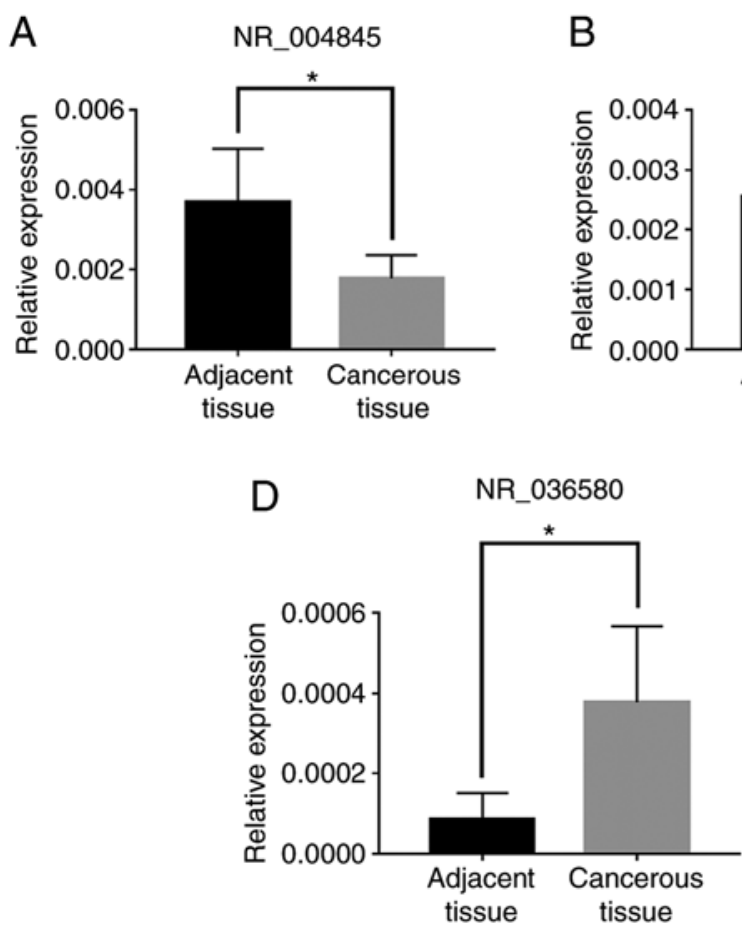
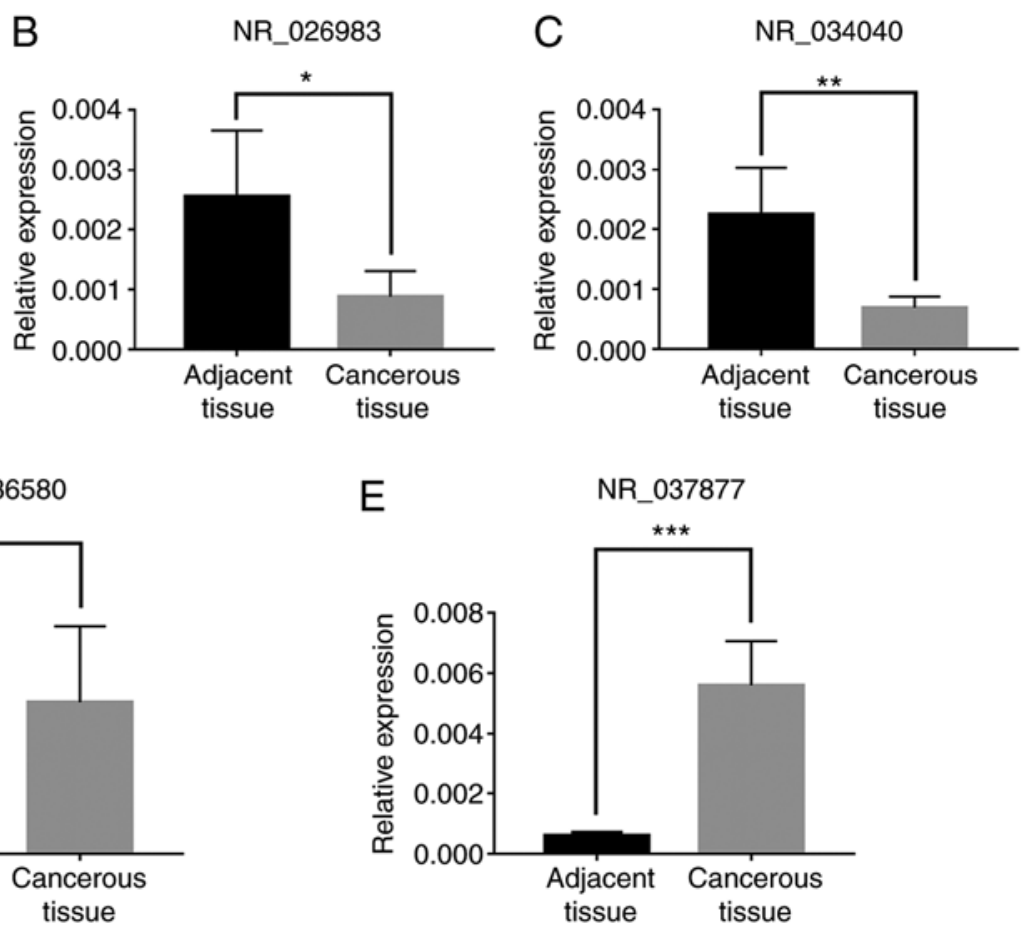

Figure 6. Validation of the microarray data by reverse transcription-quantitative PCR. The relative expression levels of differentially regulated lncRNAs are presented in ALM and adjacent non-tumor tissue: (A) NR_004845, (B) NR_026983 and (C) NR_034040 were downregulated; and (D) NR_036580 and (E) NR_037877 were upregulated in ALM. Data are presented as the mean \pm standard deviation; paired Student's t-test was used for statistical analysis. ${ }^{*} \mathrm{P}<0.05,{ }^{* *} \mathrm{P}<0.01$ and ${ }^{* * *} \mathrm{P}<0.001$ vs. adjacent non-tumor tissue. IncRNA, long non-coding RNA; ALM, acral lentiginous melanoma.

Competing endogenous network analysis, GO and KEGG analysis. The theory of competing endogenous RNA (ceRNA) has revealed that a number of ceRNAs may serve a regulatory function between coding and non-coding genes via the competition of MREs. Therefore, based on the results of the microarray analysis, a ceRNA network was constructed to determine whether lncRNAs may act as ceRNAs and contribute to the occurrence of ALM. The aforementioned five randomly selected lncRNAs were used to construct this network, which is presented in Fig. S2. The light green nodes represent lncRNAs, the red nodes represent miRNAs and the light blue nodes represent mRNAs.

GO and KEGG analyses were performed to determine the potential biological action of the ceRNA network. The results of the GO enrichment analysis demonstrated that several terms associated with tissue development, such as 'developmental process', 'anatomical structure development' and 'system development' were enriched in BP (Fig. 7D). In CC, terms such as 'cell junction' were enriched (Fig. 7E). In addition, the terms enriched in MF (Fig. 7F) were all associated with binding. These results were consistent between the microarray analysis and the ceRNA network. In KEGG analysis, pathways including 'inflammatory mediator regulation of TRP channels', 'calcium signaling pathway' and 'apelin signaling pathway' were enriched (Fig. 8B).

\section{Discussion}

No sensitive or specific biomarker for the early diagnosis and treatment of ALM is currently available. Considering the high incidence and poor outcome of ALM in Asia, especially in China, further investigations into the molecular mechanism of ALM are crucial to improve the survival rate. However, existing studies of gene mutations, epigenetics, 


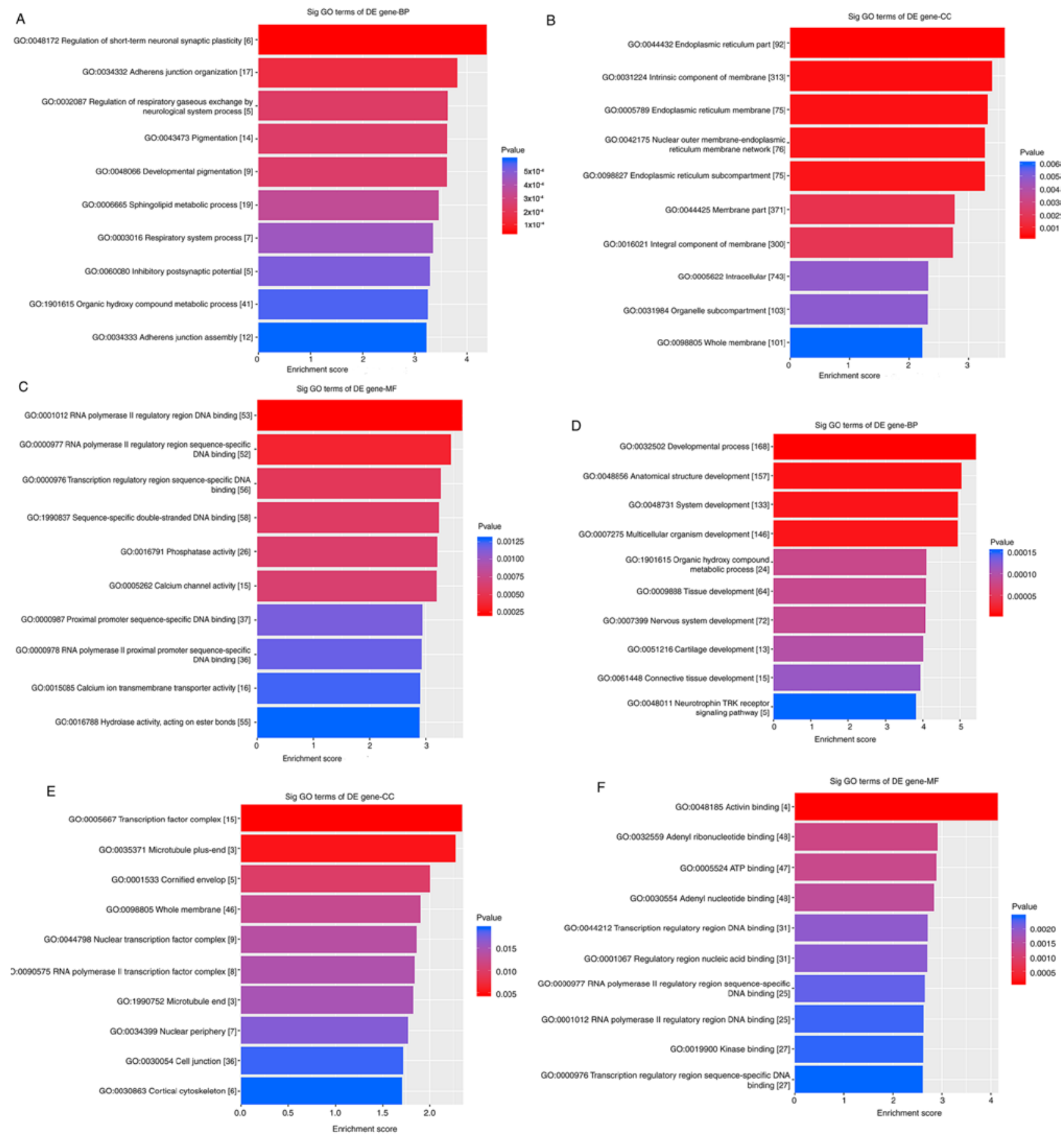

Figure 7. GO enrichment analysis in the lncRNA and mRNA co-expression network and the lncRNA/miRNA/mRNA ceRNA network. (A-C) The top 10 significantly enriched GO terms in (A) BP, (B) CC and (C) MF in the lncRNA and mRNA co-expression network. (D-F) The top 10 significantly enriched GO terms in (D) BP, (E) CC and (F) MF in the lncRNA/miRNA/mRNA ceRNA network. GO, gene ontology; lncRNA, long non-coding RNA; miRNA, microRNA; ceRNA, competing endogenous RNA; BP, biological process; CC, cellular component; MF, molecular function; DE, differentially expressed.

immune abnormalities and tumor microenvironment do not fully explain the malignant biological behaviors of ALM. The high risk of recurrence and metastasis of ALM in China has been previously attributed to patients' negligence of the disease and repeated irritation of the lesion, which is not convincing. Previously, lncRNA has emerged as a critical molecule in human cancers, such as breast and colorectal cancer, prostate, hepatocellular and basal cell carcinoma (23-26). However, a limited number of studies on lncRNAs associated with the pathogenesis, progression and metastasis of melanoma have been published and studies on ALM are lacking.

In the present study, using the microarray analysis technology, a preliminary molecular analysis of lncRNAs and mRNAs in ALM was performed to facilitate further studies on the pathogenesis of ALM and to explore whether the biological behavior of ALM may be induced by unidentified lncRNAs. In addition, GO and KEGG pathway enrichment analyses were concluded to identify the potential functions of differentially expressed mRNAs. 
A

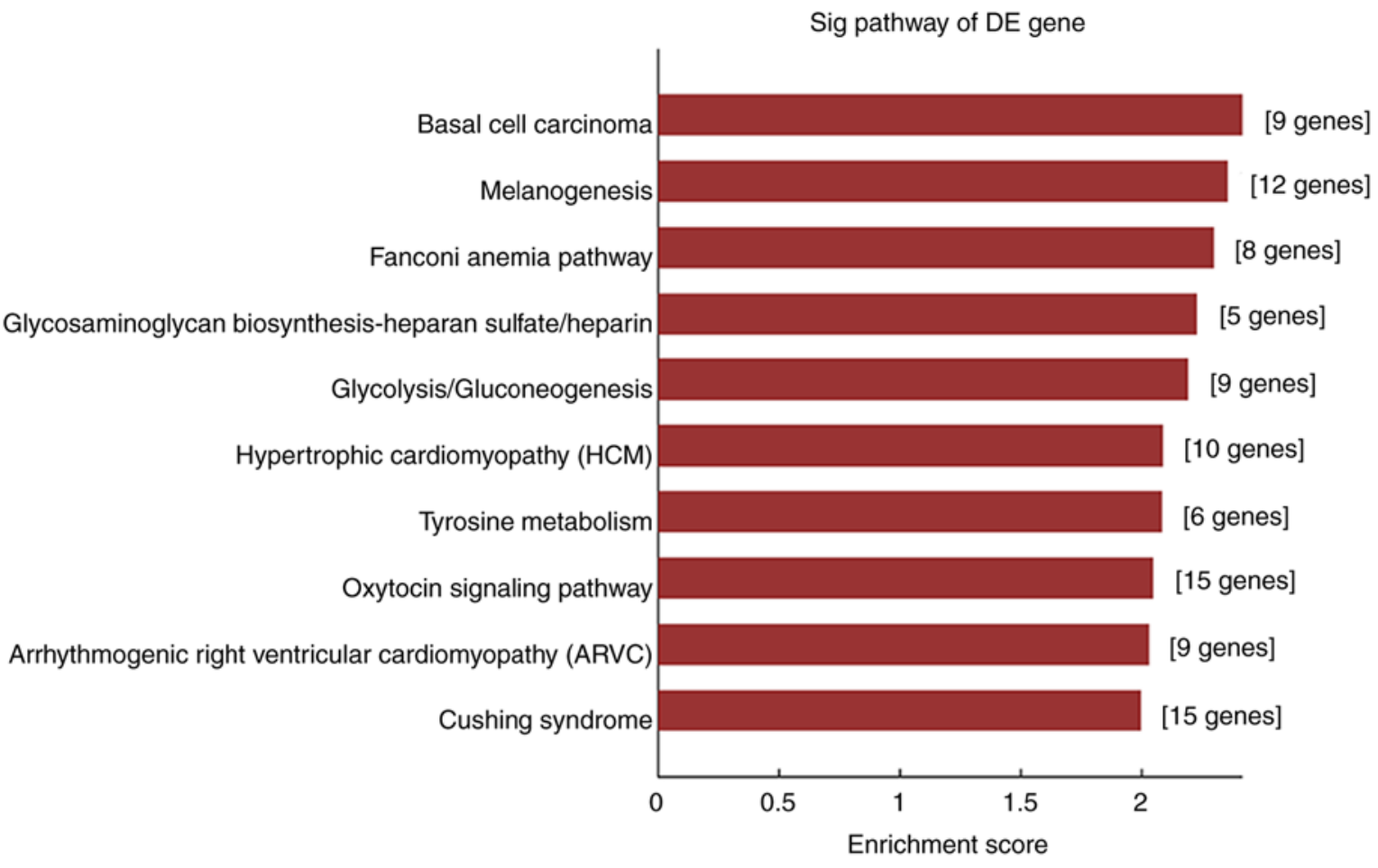

B

Sig pathway of DE gene

Histidine metabolism

Parathyroid hormone synthesis, secretion and action

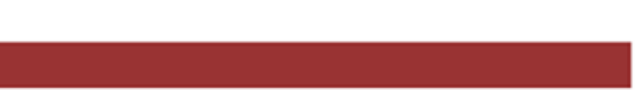

[4 genes]

Dilated cardiomyopathy (DCM)

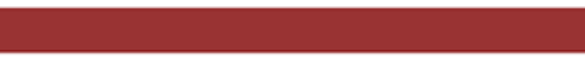

[8 genes]

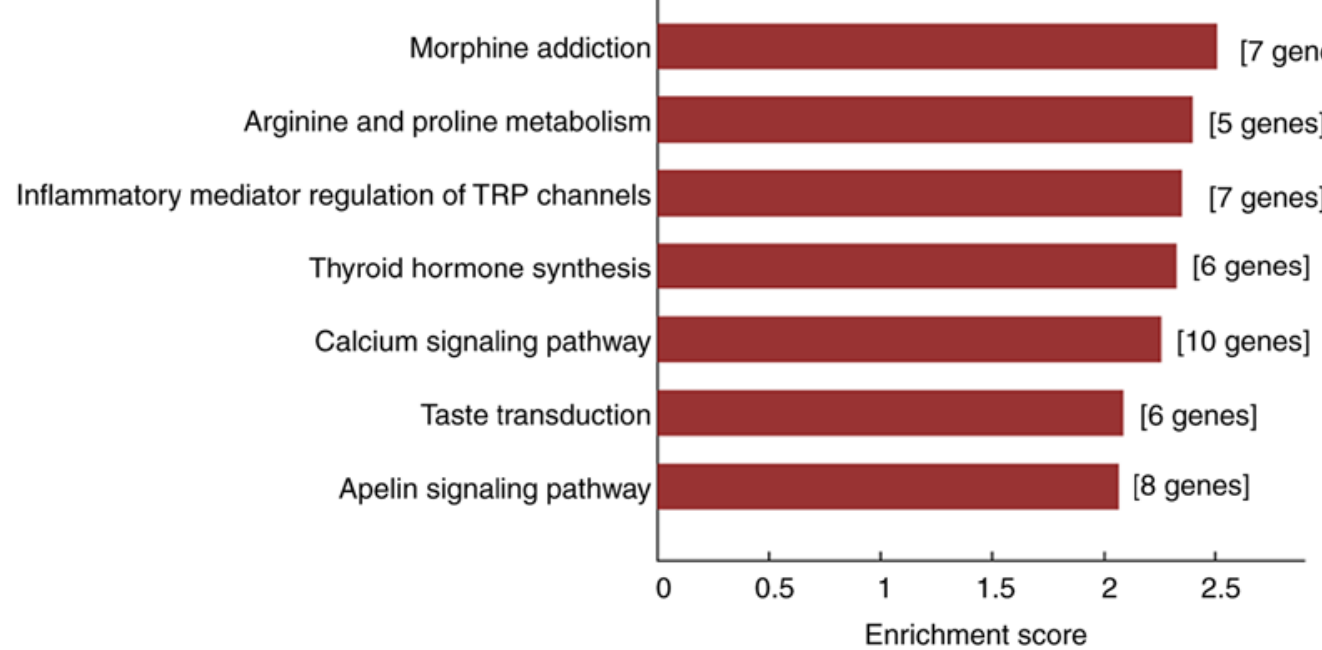

Figure 8. Kyoto Encyclopedia of Genes and Genomes pathway analysis of the lncRNA and mRNA co-expression network and the lncRNA/miRNA/mRNA ceRNA network. (A) The top 10 pathways in the lncRNA and mRNA co-expression network. (B) The top 10 pathways in the lncRNA/miRNA/mRNA ceRNA network. LncRNA, long non-coding RNA; miRNA, microRNA; ceRNA, competing endogenous RNA.

The comparison of the expression profiles of lncRNAs and mRNAs in ALM and adjacent non-tumor tissues demonstrated that 4,490 lncRNAs and 3,915 mRNAs were differentially expressed in these samples. These results were inconsistent with previous studies (8-21) of lncRNAs associated with melanoma. This may be attributed to the unique properties of ALM. The most upregulated or downregulated lncRNAs and mRNAs may help identify the molecular markers for early diagnosis of ALM. A total of five lncRNAs were randomly selected RT-qPCR; the results were consistent with those of the microarray analysis, which suggested that the results of the microarray analysis were reliable.

GO enrichment analysis was used to identify the functions of the IncRNAs through the mRNA expression patterns. Among the upregulated mRNAs, most of the BP terms were associated with pigmentation and melanocytes. Previous 
reports have demonstrated that in animal models, the inhibition of $\mathrm{Wnt} / \beta$-catenin signaling may lead to a decrease in melanocytes; however, inhibitors of the $\mathrm{Wnt} / \beta$-catenin signaling pathway do not prevent the process of pigmentation in melanoma cells $(27,28)$. One inhibitor, ICG-001, exhibited a positive effect on pigmentation (28). Another study has demonstrated that increased pigmentation is a feature of primary melanoma with a BRAF mutation; in addition, pigmentation within the sentinel node (SN) may be associated with increased SN tumor burden and prognosis (29). The presence of pigmentation may be associated with a worse clinical outcome.

The enriched CC terms were associated with organelles that promoted the formation of melanin, such as pigment granule and melanosome. These results agree with previous studies $(30,31)$. Compared with non-melanoma cells, melanoma cells are often characterized by different production of melanosomes. According to their morphology, melanosomes can be divided into four stages; stage IV suggests that the melanosome may be damaged $(30,31)$. In addition to melanin synthesis, the melanosome also serves a role in clearing toxic by-products and waste during the process of melanin synthesis; this mechanism may promote the occurrence of drug resistance (30). The mediators of drug resistance may be associated with protein products such as microphthalmia-associated transcription factor, G-protein coupled receptor 143 and premelanosome protein gp100 (31). A previous study has demonstrated that silencing the expression of genes which regulate the development of the melanosome improves the sensitivity of melanoma cells to certain drugs (30). Therefore, considering the high probability of drug resistance in ALM, further studies focusing on whether the number of melanosomes in ALM is different from other types of melanoma may be useful.

In MF, channel activity-related terms were associated with upregulated mRNAs. Previous studies have demonstrated that BKCa channels regulate cell morphology and progression, as well as the migration of tumor cells (32). In addition, the expression of $\mathrm{Na}^{+}$channels in tumor cells increase $\mathrm{Na}^{+}-\mathrm{Ca}^{2+}$ exchange, which further increases the intracellular concentration of $\mathrm{Ca}^{2+}$, enhancing the metastatic ability of tumor cells. Additionally, it also demonstrated that $\mathrm{Na}^{+}-\mathrm{Ca}^{2+}$ exchange is partly regulated by the mammalian target of rapamycin signaling pathway, which affects the proliferation and metastasis of melanoma cells (33). This is consistent with the results of the GO enrichment analysis in the present study.

For downregulated mRNAs, the BP, CC and MF terms were associated with tissue development, cell-cell junction and cell adhesion molecule binding. These terms may be associated with the progression and metastasis of ALM. Previous studies have reported that epithelial (E)-cadherin, placental (P)-cadherin and heart $(\mathrm{H})$-cadherin affect the physiological conditions of melanocytes and keratinocytes $(34,35)$. E-cadherin and $\mathrm{H}$-cadherin are often located in the basal layer of the epidermis, whereas P-cadherin is located in hair follicles. Loss of E-cadherin and expression of neural $(\mathrm{N})$-cadherin in melanoma cells are the early events in melanoma formation and metastasis (34). The expression of N-cadherin may enable melanoma cells to interact with dermal fibroblasts, which results in their migration into the dermis (35). In addition, the term 'cell adhesion' suggested that there may be numerous cell adhesion molecules associated with melanoma that may serve as targets for inhibiting growth or invasion and improving prognosis in ALM. A previous study has demonstrated that low molecular weight heparin (LMWH) inhibited the adhesion of melanoma cells through the protein kinase $\mathrm{C} \alpha(\mathrm{PKC} \alpha) / \mathrm{JNK}$ signaling pathway (36). Additionally, the integrin very late antigen-4 (VLA-4), which is a crucial molecule for the invasion of melanoma cells, is inhibited by heparin. Cyr61 also serves a role in tumor formation by activating integrin-like VLA-4. A binding site for heparin has been identified in Cyr61, thus a VLA-4/Cyr61 axis may be speculated (37). This axis may be a promising target of heparin treatment in melanoma. Nitric oxide-releasing nonsteroidal anti-inflammatory drugs inhibited the function of VLA-4 and its ligand vascular cell adhesion molecule-1, thus serving as an anti-metastasis drug for melanoma (38). Hyaluronan, which is a component of the extracellular matrix, may regulate the metastasis of melanoma through cell adhesion. Overexpression of hyaluronan synthase 3 increased the amount of hyaluronan on the cell surface and induced cell cycle arrest at G1/G0, resulting in the blockage of cell adhesion and further metastasis (39). Other molecules such as activated leukocyte cell adhesion molecule, carcinoembryonic antigen-related cell adhesion molecule 1, PRL-3/PTP4A3 phosphatase and vascular endothelial growth factor may regulate cell adhesion in melanoma and its long-term prognosis (40-43). Cell adhesion detection has also been applied in the clinical diagnosis of melanoma. The expression products of genes such as $\beta$-3 integrin, cellular tumor antigen p53, laminin $\mathrm{B} 1$ chain and tissue-type plasminogen activator may serve a role in cell adhesion and sentinel lymph node metastasis (44). Combined detection of several of the aforementioned genes may be more effective at predicting the likelihood of nodal metastasis.

KEGG pathway enrichment analysis revealed that pathways such as 'glycosphingolipid biosynthesis-ganglio series', 'toll-like receptor (TLR) signaling pathway' and 'melanogenesis' were associated with the upregulated mRNAs; these pathways have previously been demonstrated to be involved in the pathogenesis of melanoma. 'Sialyltransferase activity' is also associated with melanoma. A previous study has demonstrated that GM3 $\alpha 2,8$-sialyltransferase (GD3 synthase) served a role in the biosynthesis of gangliosides, especially GD3 (45). In melanoma, ganglioside GD3 had been identified as a tumor-specific antigen (46). The expression of the GD3 synthase gene is activated by nuclear factor $\kappa \mathrm{B}(45)$. In addition, previous studies have reported that TLR2, 3, 4, 7, 8 and 9 are expressed on melanoma cells and may interact with the development of melanoma $(47,48)$; TLR4 agonist lipopolysaccharide increases the proliferation of TLR4-positive melanoma cells. In addition, knockdown of TLR4 inhibited the migratory ability of melanoma cells (49). These results suggested that TLR4 signaling may contribute to melanoma progression.

Pathways such as 'oxytocin signaling pathway', 'regulation of actin cytoskeleton', 'hippo signaling pathway', 'focal adhesion' and 'gap junction' were associated with the downregulated mRNAs. Certain enhanced pathways, such as 'regulation of actin cytoskeleton', are also associated with LMWH (36). The inhibition of adhesion in melanoma cells through the PKC $\alpha / J N K$ signaling pathway often involves changes in the actin cytoskeleton. Another enhanced pathway 
was 'hippo signaling pathway' (50-54); most of the components of this pathway are tumor-suppressor molecules. Once the pathway is activated, phosphorylated Yes-associated protein (YAP) and paralog protein TAZ accumulate in the cell plasma and induce cell cycle arrest; when these molecules are located in the nucleus, they promote cell proliferation (50). YAP and TAZ have been identified in melanoma, and the activated hippo signaling pathway may have an inhibitory effect on the development of melanoma. The expression of TAZ in invasive melanoma is higher compared with YAP, but studies speculated that patients with melanoma with high expression of YAP tend to exhibit poor prognosis $(51,55,56)$. In addition, single nucleotide polymorphisms such as TEA domain transcription factor (TEAD) 1 and TEAD4 may also influence the survival of patients with melanoma. Therefore, these two molecules may serve as therapeutic targets for melanoma (54). A previous study has speculated that the pathogenesis of melanoma was associated with the crosstalk between hippo and mitogen associated protein kinase signaling pathways via the interaction of Raf-1 proto-oncogene serine/threonine kinase and serine/threonine kinase 3 (52).

LncRNAs affect the pathogenesis of various diseases through epigenetic regulation. To determine the exact mechanism of lncRNAs involved in ALM, a ceRNA network between lncRNAs, miRNAs and mRNAs was constructed as lncRNAs may disturb the activity of certain miRNAs, which would subsequently affect their target mRNAs. In this ceRNA network, five lncRNAs interacted with 417 mRNAs through 252 miRNAs. The results of GO and KEGG analysis were similar to those of the genes identified by microarray analysis.

Limitations existed in our study: What has been done in the present study was just a microarray analysis and its related CNC network, ceRNA network, GO analysis and KGEG analysis based on the predicted targeted genes. The further research of IncRNA function would be performed in the authors' future investigation and perhaps at that time, more evidence would be found.

In conclusion, to the best of our knowledge, the present study is the first to reveal lncRNA expression patterns in ALM using microarray analysis. The results of the present study suggested genes implicated in tissue development, pigmentation, cell adhesion activity, organelles related to melanin formation and channel activity may be involved in the pathogenesis and metastasis of ALM. In addition, the CNC and ceRNA network analysis results suggested that dysregulated lncRNAs and mRNAs may serve a role in tumor formation and development, and lncRNAs may also act as ceRNAs to disturb the pathogenesis of ALM. These molecules may be promising therapeutic targets for patients with ALM and further studies are needed to explore the precise mechanisms of ALM.

\section{Acknowledgements}

Not applicable.

\section{Funding}

The present study was supported by grants from the CAMS Innovation Fund for Medical Sciences (CIFMS-2017-12M-1-017) and the PUMC Youth Fund (grant no. 3332017168).

\section{Availability of data and materials}

The datasets used and/or analyzed during the current study are available from the corresponding author on reasonable request.

\section{Authors' contributions}

HS, JX, HC, YW and JS conceived this study. HS, JX, CX and WB performed the experiments and collected the data. Data were analyzed and interpreted by $\mathrm{HC}, \mathrm{YW}$ and JS. HS wrote the paper. The paper was reviewed by HC, YW and JS. All authors have read and approved the manuscript.

\section{Ethics approval and consent to participate}

The present study was approved by the Ethics Committee of Institute ofDermatology, Chinese Academy of Medical Sciences and Peking Union Medical College (2013-LC/KY-033). All patients provided informed consents.

\section{Patient consent for publication}

Not applicable.

\section{Competing interests}

The authors declare that they have no competing interests.

\section{References}

1. Lino-Silva LS, Zepeda-Najar C, Salcedo-Hernández RA and Martinez-Said H: Acral lentiginous melanoma: Survival analysis of 715 cases. J Cutan Med Surg 23: 38-43, 2019.

2. Häfliger EM, Ramelyte E, Mangana J, Kunz M, Kazakov DV, Dummer R and Cheng PF: Metastatic acral lentiginous melanoma in a tertiary referral center in Switzerland: A systematic analysis. Melanoma Res 28: 442-450, 2018.

3. Kim HJ, Seo JW, Roh MS, Lee JH and Song KH: Clinical features and prognosis of Asian patients with acral lentiginous melanoma who have nodal nevi in their sentinel lymph node biopsy specimen. J Am Acad Dermatol 79: 706-713, 2018.

4. Nakamura Y and Fujisawa Y: Diagnosis and management of acral lentiginous melanoma. Curr Treat Options Oncol 19: 42, 2018.

5. Wada M, Ito T, Tsuji G, Nakahara T, Hagihara A, Furue M and Uchi H: Acral lentiginous melanoma versus other melanoma: A single-center analysis in Japan. J Dermatol 44: 932-938, 2017.

6. Hombach S and Kretz M: Non-coding RNAs: Classification, biology and functioning. Adv Exp Med Biol 937: 3-17, 2016.

7. Kondo Y, Shinjo K and Katsushima K: Long non-coding RNAs as an epigenetic regulator in human cancers. Cancer Sci 108: 1927-1933, 2017.

8. Yu X, Zheng H, Tse G, Chan MT and Wu WK: Long non-coding RNAs in melanoma. Cell Prolif 51: e12457, 2018.

9. Tang L, Zhang W, Su B and Yu B: Long noncoding RNA HOTAIR is associated with motility, invasion, and metastatic potential of metastatic melanoma. Biomed Res Int 2013: 251098, 2013.

10. Sun L, Sun P, Zhou QY, Gao X and Han Q: Long noncoding RNA MALAT1 promotes uveal melanoma cell growth and invasion by silencing of miR-140. Am J Transl Res 8: 3939-3946, 2016.

11. Flockhart RJ, Webster DE, Qu K, Mascarenhas N, Kovalski J, Kretz M and Khavari PA: BRAFV600E remodels the melanocyte transcriptome and induces BANCR to regulate melanoma cell migration. Genome Res 22: 1006-1014, 2012.

12. Li R, Zhang L, Jia L, Duan Y, Li Y, Bao L and Sha N: Long non-coding RNA BANCR promotes proliferation in malignant melanoma by regulating MAPK pathway activation. PLoS One 9: e100893, 2014. 
13. Xu S, Wang H, Pan H, Shi Y, Li T, Ge S, Jia R, Zhang H and Fan X: ANRIL lncRNA triggers efficient therapeutic efficacy by reprogramming the aberrant INK4-hub in melanoma. Cancer Lett 381: 41-48, 2016.

14. Pasmant E, Laurendeau I, Héron D, Vidaud M, Vidaud D and Bièche I: Characterization of a germ-line deletion, including the entire INK4/ARF locus, in a melanoma-neural system tumor family: Identification of ANRIL, an antisense noncoding RNA whose expression coclusters with ARF. Cancer Res 67: 3963-3969, 2007.

15. Mazar J, Zhao W, Khalil AM, Lee B, Shelley J, Govindarajan SS, Yamamoto F, Ratnam M, Aftab MN, Collins S, et al: The functional characterization of long noncoding RNA SPRY4-IT1 in human melanoma cells. Oncotarget 5: 8959-8969, 2014

16. Khaitan D, Dinger ME, Mazar J, Crawford J, Smith MA, Mattick JS and Perera RJ: The melanoma-upregulated long noncoding RNA SPRY4-IT1 modulates apoptosis and invasion. Cancer Res 71: 3852-3862, 2011.

17. Wu CF, Tan GH, Ma CC and Li L: The non-coding RNA llme23 drives the malignant property of human melanoma cells. J Genet Genomics 40: 179-188, 2013.

18. Wei Y, Sun Q, Zhao L, Wu J, Chen X, Wang Y, Zang W and Zhao G: LncRNA UCA1-miR-507-FOXM1 axis is involved in cell proliferation, invasion and G0/G1 cell cycle arrest in melanoma. Med Oncol 33: 88, 2016

19. Tian Y, Zhang X, Hao Y, Fang Z and He Y: Potential roles of abnormally expressed long noncoding RNA UCA1 and Malat-1 in metastasis of melanoma. Melanoma Res 24: 335-341, 2014.

20. Schmidt K, Joyce CE, Buquicchio F, Brown A, Ritz J, Distel RJ, Yoon $\mathrm{CH}$ and Novina CD: The lncRNA SLNCR1 mediates melanoma invasion through a conserved SRA1-like region. Cell Rep 15: 2025-2037, 2016.

21. LeucciE,Vendramin R,Spinazzi M,LauretteP,Fiers M, Wouters J, Radaelli E, Eyckerman S, Leonelli C, Vanderheyden K, et al: Melanoma addiction to the long non-coding RNA SAMMSON. Nature 531: 518-522, 2016

22. Larionov A, Krause A and Miller W: A standard curve based method for relative real time PCR data processing. BMC Bioinformatics 6: 62, 2005 .

23. Forrest ME and Khalil AM: Review: Regulation of the cancer epigenome by long non-coding RNAs. Cancer Lett 407: 106-112, 2017.

24. Xu N, Wang F, Lv M and Cheng L: Microarray expression profile analysis of long non-coding RNAs in human breast cancer: A study of Chinese women. Biomed Pharmacother 69: 221-227, 2015.

25. Sand M, Bechara FG, Sand D, Gambichler T, Hahn SA, Bromba M, Stockfleth E and Hessam S: Long-noncoding RNAs in basal cell carcinoma. Tumour Biol 37: 10595-10608, 2016.

26. Xue Y, Ma G, Gu D, Zhu L, Hua Q, Du M, Chu H, Tong N, Chen J, Zhang $\mathrm{Z}$ and Wang M: Genome-wide analysis of long noncoding RNA signature in human colorectal cancer. Gene 556: 227-234, 2015.

27. Dorsky RI, Moon RT and Raible DW: Control of neural crest cell fate by the Wnt signalling pathway. Nature 396: 370-373, 1998.

28. Kim KI, Jeong DS, Jung EC, Lee JH, Kim CD and Yoon TJ: $\mathrm{Wnt} / \beta$-catenin signaling inhibitor ICG-001 enhances pigmentation of cultured melanoma cells. J Dermatol Sci 84: 160-168, 2016.

29. van Lanschot CG, Koljenovic S, Grunhagen DJ, Verhoef $\mathrm{C}$ and van Akkooi AC: Pigmentation in the sentinel node correlates with increased sentinel node tumor burden in melanoma patients. Melanoma Res 24: 261-266, 2014.

30. Chen KG, Leapman RD, Zhang G, Lai B, Valencia JC, Cardarelli CO, Vieira WD, Hearing VJ and Gottesman MM: Influence of melanosome dynamics on melanoma drug sensitivity. J Natl Cancer Inst 101: 1259-1271, 2009

31. Hertzman Johansson C, Azimi A, Frostvik Stolt M, Shojaee S, Wiberg H, Grafström E, Hansson J and Egyházi Brage S: Association of MITF and other melanosome-related proteins with chemoresistance in melanoma tumors and cell lines. Melanoma Res 23: 360-365, 2013.

32. Tajima N, Itokazu Y, Korpi ER, Somerharju P and Käkelä R: Activity of $\mathrm{BK}(\mathrm{Ca})$ channel is modulated by membrane cholesterol content and association with $\mathrm{Na}+\mathrm{K}+-\mathrm{ATPa} e$ in human melanoma IGR39 cells. J Biol Chem 286: 5624-5638, 2011.

33. Yang Y, Luo Z, Hao Y, Ba W, Wang R, Wang W, Ding X and $\mathrm{Li}$ C: mTOR-mediated $\mathrm{Na}^{+} / \mathrm{Ca}^{2+}$ exchange affects cell proliferation and metastasis of melanoma cells. Biomed Pharmacother 92: 744-749, 2017.
34. Kuphal S and Bosserhoff AK: E-cadherin cell-cell communication in melanogenesis and during development of malignant melanoma. Arch Biochem Biophys 524: 43-47, 2012.

35. Rossier-Pansier L, Baruthio F, Rüegg C and Mariotti A: Compartmentalization in membrane rafts defines a pool of $\mathrm{N}$-cadherin associated with catenins and not engaged in cell-cell junctions in melanoma cells. J Cell Biochem 103: 957-971, 2008.

36. Chalkiadaki G, Nikitovic D, Katonis P, Berdiaki A, Tsatsakis A Kotsikogianni I, Karamanos NK and Tzanakakis GN: Low molecular weight heparin inhibits melanoma cell adhesion and migration through a $\mathrm{PKCa} / \mathrm{JNK}$ signaling pathway inducing actin cytoskeleton changes. Cancer Lett 312: 235-244, 2011.

37. Schmitz P, Gerber U, Schütze N, Jüngel E, Blaheta R, Naggi A, Torri $\mathrm{G}$ and Bendas G: Cyr61 is a target for heparin in reducing MV3 melanoma cell adhesion and migration via the integrin VLA-4. Thromb Haemost 110: 1046-1054, 2013.

38. Cheng H, Mollica MY, Lee SH, Wang L, Velazquez-Martinez CA and $\mathrm{Wu} \mathrm{S}$ : Effects of nitric oxide-releasing nonsteroidal anti-inflammatory drugs (NONO-NSAIDs) on melanoma cell adhesion. Toxicol Appl Pharmacol 264: 161-166, 2012.

39. Takabe P, Bart G, Ropponen A, Rilla K, Tammi M, Tammi R and Pasonen-Seppänen S: Hyaluronan synthase 3 (HAS3) overexpression downregulates MV3 melanoma cell proliferation, migration and adhesion. Exp Cell Res 337: 1-15, 2015.

40. Valcárcel M, Mendoza L, Hernández JJ, Carrascal T, Salado C, Crende $\mathrm{O}$ and Vidal-Vanaclocha F: Vascular endothelial growth factor regulates melanoma cell adhesion and growth in the bone marrow microenvironment via tumor cyclooxygenase-2. J Transl Med 9: 142, 2011.

41. Foy M, Anézo O, Saule S and Planque N: PRL-3/PTP4A3 phosphatase regulates integrin $\beta 1$ in adhesion structures during migration of human ocular melanoma cells. Exp Cell Res 353: 88-99, 2017.

42. van Kilsdonk JW, Takahashi N, Weidle U, Burtscher H, Jarry J, Daha MR, Swart GW and van Kempen LC: Modulation of activated leukocyte cell adhesion molecule-mediated invasion triggers an innate immune gene response in melanoma. J Invest Dermatol 132: 1462-1470, 2012.

43. Ullrich N, Löffek S, Horn S, Ennen M, Sánchez-Del-Campo L, Zhao F, Breitenbuecher F, Davidson I, Singer BB, Schadendorf D, et al: MITF is a critical regulator of the carcinoembryonic antigen-related cell adhesion molecule 1 (CEACAM1) in malignant melanoma. Pigment Cell Melanoma Res 28: 736-740, 2015.

44. Meves A, Nikolova E, Heim JB, Squirewell EJ, Cappel MA, Pittelkow MR, Otley CC, Behrendt N, Saunte DM, Lock-Andersen J, et al: Tumor cell adhesion as a risk factor for sentinel lymph node metastasis in primary cutaneous melanoma. J Clin Oncol 33: 2509-2515, 2015.

45. Kang NY, Kim CH, Kim KS, Ko JH, Lee JH, Jeong YK and Lee YC: Expression of the human CMP-NeuAc:GM3 alpha2,8-sialyltransferase (GD3 synthase) gene through the NF-kappaB activation in human melanoma SK-MEL-2 cells. Biochim Biophys Acta 1769: 622-630, 2007.

46. Miyata M, Ichihara M, Tajima O, Sobue S, Kambe M, Sugiura K, Furukawa K and Furukawa K: UVB-irradiated keratinocytes induce melanoma-associated ganglioside GD3 synthase gene in melanocytes via secretion of tumor necrosis factor $\alpha$ and interleukin 6. Biochem Biophys Res Commun 445: 504-510, 2014.

47. Goto Y, Arigami T, Kitago M, Nguyen SL, Narita N, Ferrone S, Morton DL, Irie RF and Hoon DS: Activation of Toll-like receptors 2,3 , and 4 on human melanoma cells induces inflammatory factors. Mol Cancer Ther 7: 3642-3653, 2008.

48. Saint-Jean M, Knol AC, Nguyen JM, Khammari A and Dréno B: TLR expression in human melanoma cells. Eur J Dermatol 21: 899-905, 2011.

49. Feng R, Gong J, Wu L, Wang L, Zhang B, Liang G, Zheng H and Xiao H: MAPK and Hippo signaling pathways crosstalk via the RAF-1/MST-2 interaction in malignant melanoma. Oncol Rep 38: 1199-1205, 2017.

50. Kim JE, Finlay GJ and Baguley BC: The role of the hippo pathway in melanocytes and melanoma. Front Oncol 3: 123, 2013.

51. Menzel M,MeckbachD, Weide B,ToussaintNC,SchilbachK, NoorS, Eigentler T, Ikenberg K, Busch C, Quintanilla-Martinez L, et al: In melanoma, Hippo signaling is affected by copy number alterations and YAP1 overexpression impairs patient survival. Pigment Cell Melanoma Res 27: 671-673, 2014.

52. Nallet-Staub F, Marsaud V, Li L, Gilbert C, Dodier S, Bataille V, Sudol M, Herlyn M and Mauviel A: Pro-invasive activity of the Hippo pathway effectors YAP and TAZ in cutaneous melanoma. J Invest Dermatol 134: 123-132, 2014. 
53. Takazawa Y, Kiniwa Y, Ogawa E, Uchiyama A, Ashida A, Uhara $\mathrm{H}$, Goto $\mathrm{Y}$ and Okuyama R: Toll-like receptor 4 signaling promotes the migration of human melanoma cells. Tohoku J Exp Med 234: 57-65, 2014.

54. Yuan H, Liu H, Liu Z, Zhu D, Amos CI, Fang S, Lee JE and Wei Q: Genetic variants in Hippo pathway genes YAP1, TEAD1 and TEAD4 are associated with melanoma-specific survival. Int J Cancer 137: 638-645, 2015

55. Hall CA, Wang R, Miao J, Oliva E, Shen X, Wheeler T, Hilsenbeck SG, Orsulic S and Goode S: Hippo pathway effector Yap is an ovarian cancer oncogene. Cancer Res 70: 8517-8525, 2010.
56. Kang W, Tong JH, Chan AW, Lee TL, Lung RW, Leung PP, So KK, Wu K, Fan D, Yu J, et al: Yes-associated protein 1 exhibits oncogenic property in gastric cancer and its nuclear accumulation associates with poor prognosis. Clin Cancer Res 17: 2130-2139, 2011.

(i) () $\odot$ This work is licensed under a Creative Commons EY NG ND Attribution-NonCommercial-NoDerivatives 4.0 International (CC BY-NC-ND 4.0) License. 